\title{
Long-period oscillations of millimeter emission above sunspots
}

\author{
V. Smirnova ${ }^{1,2}$, A. Riehokainen ${ }^{1}$, V. Ryzhov ${ }^{3}$, A. Zhiltsov ${ }^{3}$, and J. Kallunki ${ }^{1,4}$ \\ ${ }^{1}$ University of Turku, 20014 Turku, Finland \\ e-mail: vvsvid@rambler.ru \\ 2 Sobolev Astronomical Institute, Saint Petersburg State University, Universitetsky pr.28, St. Petergof 198504, Russia \\ 3 Bauman Moscow State Technical University, 2 Bauman street 5, Moscow 105005, Russia \\ 4 Aalto University, Metsähovi Radio Observatory, Kylmälä, Finland
}

Received 15 June 2011 / Accepted 1 September 2011

\section{ABSTRACT}

\begin{abstract}
Aims. The investigation of long quasi-periodic oscillations in sunspots/active regions based on millimeter radio data was the main goal of this work.

Methods. Data from simultaneous monitoring observations of solar active regions at $37 \mathrm{GHz}$ and $93 \mathrm{GHz}$ at two different groundbased radio telescopes were obtained. We analyzed them with the methods of wavelet (Morlet) and global wavelet spectrum analysis. Results. Two main ranges (10-60 and 80-130 min) of long quasi-periodic oscillations were found. We compared them with $17 \mathrm{GHz}$ Nobeyama Radioheliograph (NoRH) data and obtained the same ranges for the long-period oscillations. The long-period oscillations found in this study are relatively stable and could be interpreted as a radial mode of sunspot oscillations.
\end{abstract}

Key words. Sun: oscillations - Sun: radio radiation

\section{Introduction}

The investigation of solar oscillations is a good method to analyze the physical properties and the spatial structure of active regions at all wavelengths. But at radio wavelengths it is of special interest because of the possibility to obtain unique information about the plasma parameters at heights corresponding to the chromosphere, the transition region, and the corona.

The solar oscillatory phenomenon has been investigated by many authors for several decades (for example: Kobrin \& Korshunov 1972; Thomas et al. 1984; Gelfreikh et al. 1999; Bogdan 2000; Nagovitsyna \& Nagovitsyn 2001; Nakariakov 2007; Solov'ev \& Kirichek 2008; Abramov-Maximov et al. 2011; Kislyakova et al. 2011). A good review of solar oscillations is presented in Kosovichev (2009). A significant number of reports have been devoted to short-period oscillations (3-5 min). But long-period oscillations (30 min and more) of active regions and sunspots have been studied considerably less than the 3-5 min oscillations. The mechanism responsible for the generation of the long-period component in sunspot oscillations is under discussion. These oscillations have been observed in the variation of the magnetic-field strength and the line-of-sight velocity (Efremov et al. 2010). The theoretical interpretation of long quasi-periodic oscillations in sunspots is related to the so-called "shallow" model of sunspots, suggested by Solov'ev $\&$ Kirichek (2008). This model presents the sunspot as a stable structure, separated from the other environment, which can quasi-periodically change its magnetic-field strength and the geometrical size (Efremov et al. 2007).

Also, the low-frequency variations of the microwave radio emission above spots were analyzed, for example in Gelfreikh et al. (2006), Chorley et al. (2010), and Khodachenko et al. (2011). But the theoretical interpretation of long-period oscillations above sunspots in radio emission is not clear.
Simultaneous monitoring observations of active regions at short radio frequencies, especially at millimeter wavelength, is an actual goal for the present time, because it gives the possibility to obtain the information about the source structure and parameters at different chromospheric heights. However, it is difficult to obtain good-quality millimeter data. Investigations of the millimeter emission are rare and face a lot of problems such as instrument limitations, terrestrial atmospheric fluctuations, and the signal absorption on the propagation track (Nagnibeda \& Piotrovich 1994). There are only a few instruments for solar observations that have the capability to work in the millimeter range today. In this paper we present the millimeter data analysis obtained from the RT-7.5 radio-telescope belonging to the Bauman Moscow State Technical University (BMSTU, Moscow Region, Russia) and the RT-14 Metsähovi radio-telescope (Aalto University, Helsinki Region, Finland) and also, for comparison, a microwave data analysis from the Nobeyama Radioheliograph (Japan). The methods of observations and calibrations are described in Sect. 2, data analysis and results are presented in Sect. 3, and finally the discussion and conclusions are presented in Sect. 4.

\section{Observations}

\subsection{Instruments: RT-7.5 BMSTU}

The RT-7.5 radio-telescope is a Cassegrain-type antenna with a diameter of $7.75 \mathrm{~m}$. It has two receivers for $93 \mathrm{GHz}$ and $140 \mathrm{GHz}$ (3.2 $\mathrm{mm}$ and $2.2 \mathrm{~mm}$ ). The beam size of the telescope is $2.5^{\prime}$ and $1.5^{\prime}$, respectively. We used only the $93 \mathrm{GHz}$ receiver for solar observations. The sensitivity of the receiver is $0.3 \mathrm{~K}$ with the time constant of $1 \mathrm{~s}$.

The antenna can provide both whole disk mapping and tracking of a selected point. The maximum temporal resolution 
Table 1. Results obtained from RT-7.5 and RT-14 during the observational period: 2010 June 24-2010 August 31.

\begin{tabular}{lccc}
\hline \hline $\begin{array}{l}\text { Date and notes } \\
\text { (TS - simultaneous tracks; }\end{array}$ & NOAA/Area & Frequency $(\mathrm{GHz})$ & Long periods (10 min and more) \\
TR - RT-7.5; TF - RT-14; & & & \\
\hline $2010-07-01$ TS; & $11084 / 0110$ & 37,93 & $10-60 ; 80-130$ \\
$2010-07-02$ TR; & $11084 / 0110$ & 93 & $16-50 ; 70-130$ \\
$2010-07-05$ TR; & $11084 / 0110$ & 93 & $20-40 ; 70-80$ \\
$2010-07-28$ TR; & $11089 / 0070$ & 93 & $70-80$ \\
$2010-08-05$ TR; & $11092 / 0200$ & 93 & $40-60$ \\
$2010-08-19$ TS; & $11100 / 0010$ & 37,93 & $10-30 ; 40-130$ \\
\hline
\end{tabular}

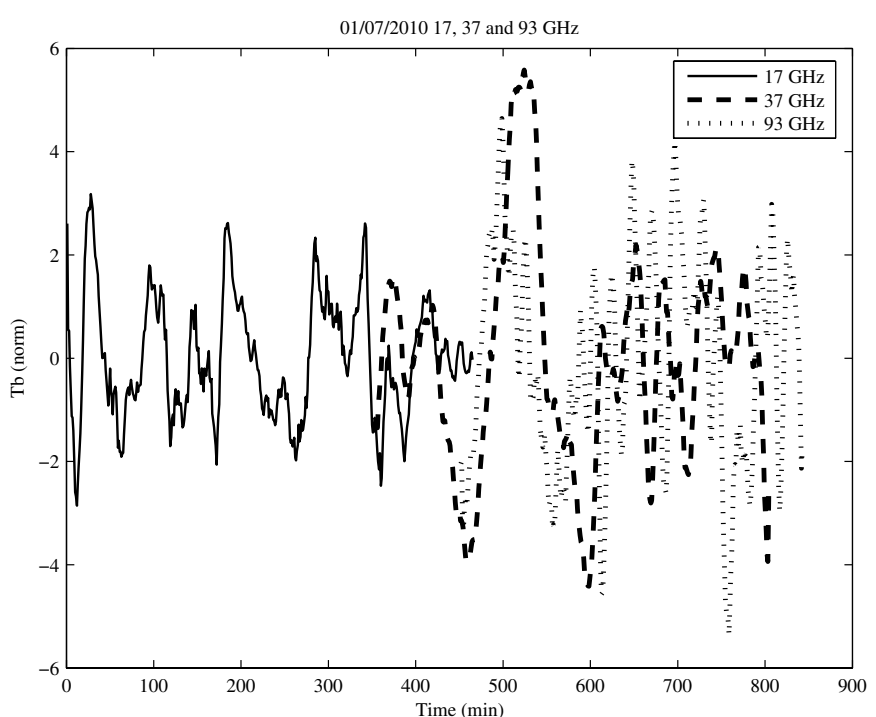

Fig. 1. Tracks for 17,37, and $93 \mathrm{GHz}$ data calculated for the normalized brightness temperature on 2010 July 1, AR 11084.

is $0.125 \mathrm{~s}$ and the noise in the obtained signal is $1-1.5 \%$. The maximum error of the beam pointing is $10^{\prime \prime}$ (Rozanov 1981; Efremov \& Rozanov 2001).

\subsection{Instruments: $R T-14$ Metsähovi}

The Metsähovi RT-14 radio-telescope is a Cassegrain-type antenna with a diameter of $13.7 \mathrm{~m}$. The working range of the telescope is $2-150 \mathrm{GHz}(13.0 \mathrm{~cm}-2.0 \mathrm{~mm})$. The antenna provides both solar mapping and active region tracking. In this study only the $37 \mathrm{GHz}$ receiver was used for solar observations. The beam size of the telescope is $2.4^{\prime}$. The sensitivity of the receiver is sufficient for 0.2 s.f.u. resolution. The temporal resolution is $0.1 \mathrm{~s}$ and the method of active region observations is the continuous tracking (Urpo 1975).

\section{Data analysis and results}

We obtained several sessions of observations of selected active regions with durations of $6-8 \mathrm{~h}$ to detect long period oscillations above sunspots. The choice of long periods was made because this type of oscillations at microwave and millimeter waves does not have accepted theoretical interpretations.

The results presented in Table 1 were obtained at RT-7.5 and RT-14 during the observational period from 2010 June 24 to 2010 August 31. Because of the difference in weather conditions, we obtained only two simultaneous sessions, on 2010 July 1 and 2010 August 19.

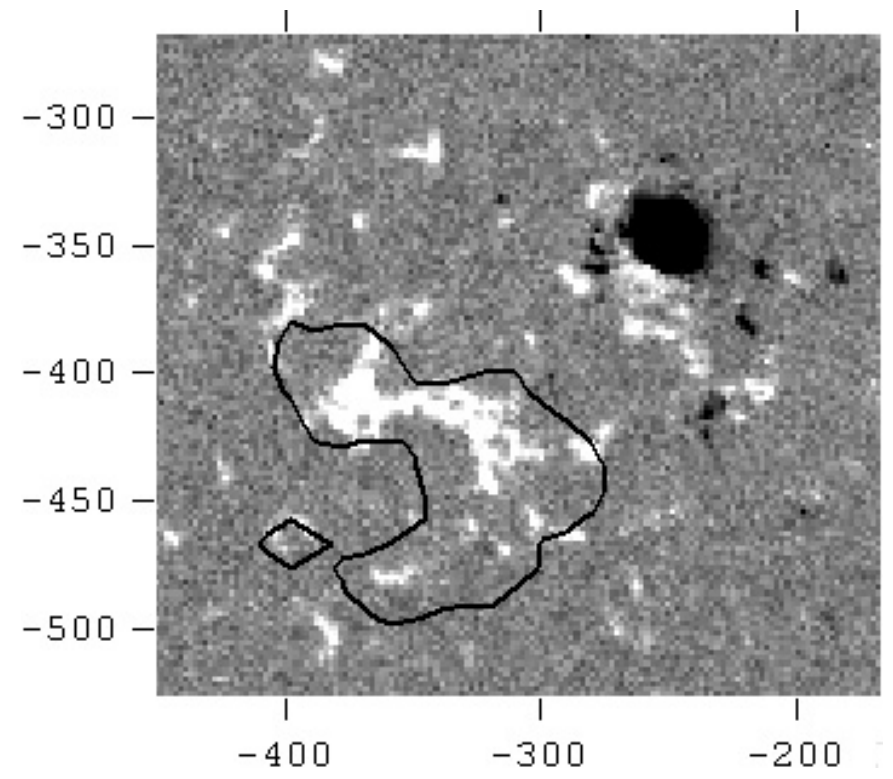

Fig. 2. SOHO/MDI magnetogram (Bmax is about 2350 Gauss) with superposed $17 \mathrm{GHz}$ source contours at the brightness temperature level of $11000 \mathrm{~K}$ on 2010 July 1, AR 11084.

Additionally, to compare our results obtained at $37 \mathrm{GHz}$ and $93 \mathrm{GHz}$ with high spatial resolution data, we analyzed solar radio maps observed with the Nobeyama Radioheliograph at $17 \mathrm{GHz}$ (Nakajima et al. 1994). For that, we synthesized images by the Koshix algorithm using the SolarSoft with the averaging of $1 \mathrm{~min}$. We also integrated the source area on the region of size of $2.5^{\prime}$ to provide the comparison with data obtained by the wide beam.

The process of data analysis we demonstrate with data obtained on 2010 July 1. Tracks for this day are shown in Fig. 1. These tracks were obtained for the active region (AR) 11084. This figure also shows the Nobeyama Radioheliograph track $(17 \mathrm{GHz})$, marked in the plot by the solid black curve, for the same active area, but observed some hours earlier than observations at $37 \mathrm{GHz}$ and $93 \mathrm{GHz}$. The large magnetic structure (sunspot) existed during a long period of time and is also visible in the SOHO/MDI magnetogram (Fig. 2). The center of the antenna beam for both radio-telescopes coincided with the distributed positive magnetic polarity structures surrounded by the contours of $17 \mathrm{GHz}$ NoRH source seen in Fig. 2.

The data processing consisted of the following stages:

1. We defined trends in the records that were related with the absorption coefficient, which changed during the time of observation.

2. After the trend analysis we removed it by a fourth order polynomial function. 

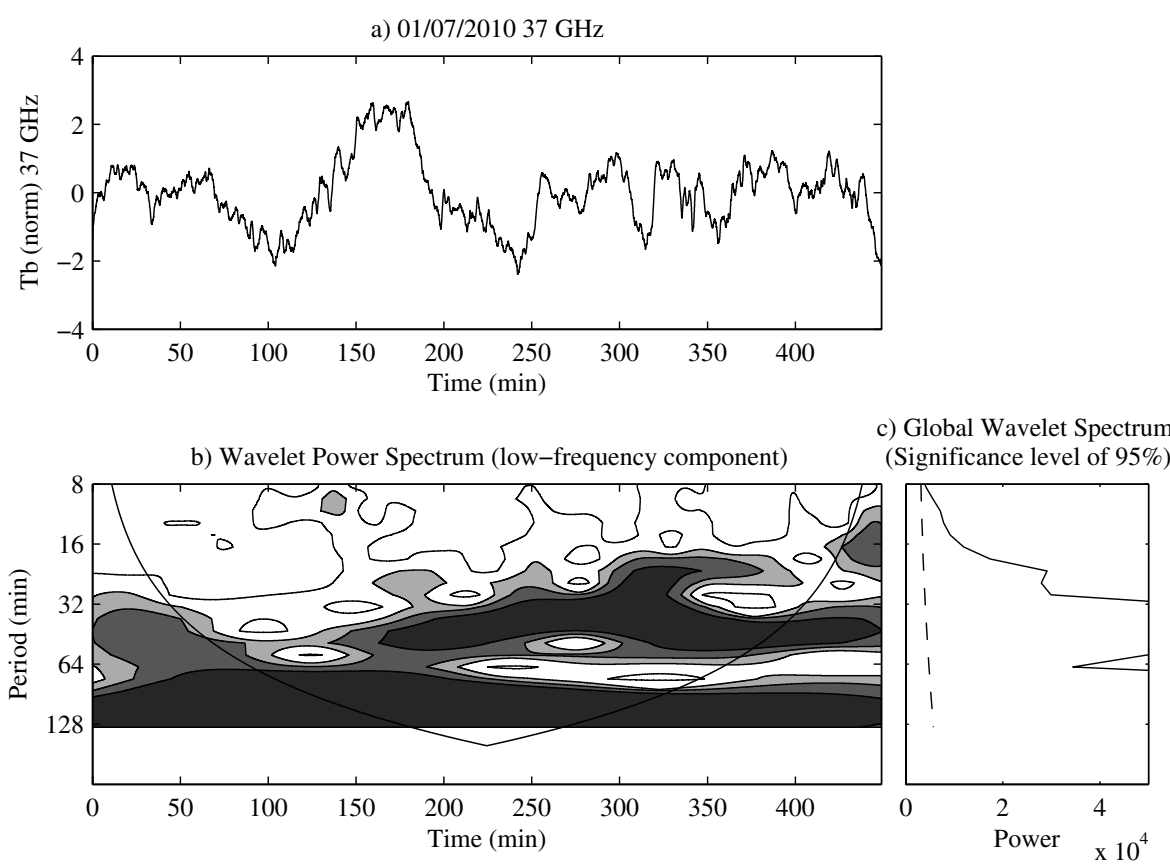

Fig. 3. Track (panel a)), the low-frequency component of the wavelet spectrum (panel b)), the global wavelet spectrum with $95 \%$ significance level (panel c)) for $37 \mathrm{GHz}$ data on 2010 July 1 for the source AR 11084.

a) $01 / 07 / 201093 \mathrm{GHz}$

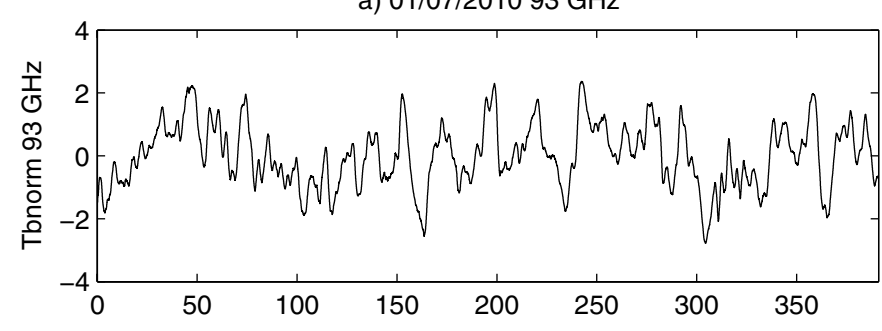

c) Global Wavelet Spectrun b) Wavelet Power Spectrum (low-frequency component) (Significance level of $95 \%$ )

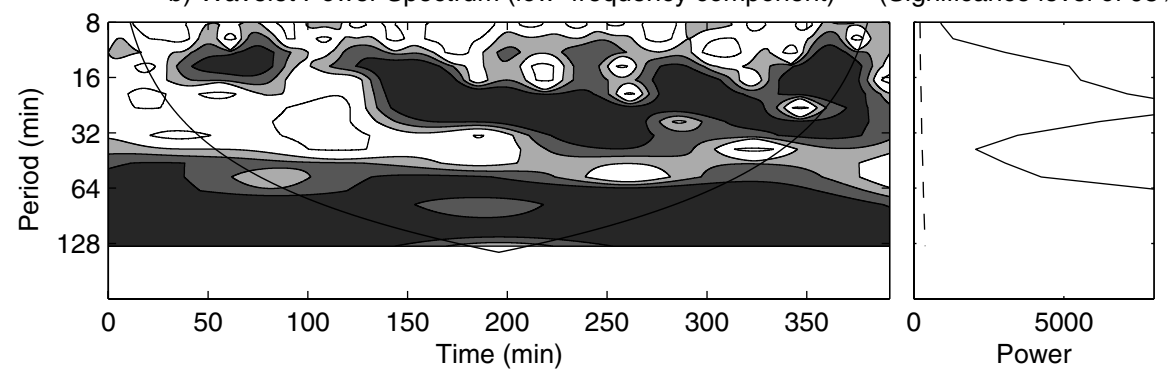

Fig. 4. Same as in Fig. 3 for $93 \mathrm{GHz}$ data on 2010 July 1 for the source AR 11084.

3. We searched for the long quasi-periodic oscillations with the Wavelet method.

To compute the wavelet power spectrum, we used the technique described by Torrence \& Compo (1998).

Because we were interested in long-period oscillations, we chose the corresponding scales for the wavelet and created power spectra for $37 \mathrm{GHz}$ and $93 \mathrm{GHz}$ data (the same was made for the $17 \mathrm{GHz}$ data, which were taken for comparison). Powerful long quasi-periodic oscillations were detected in radio emission at $37 \mathrm{GHz}$ mainly in two different period intervals (16-60 and 80-130 min). They are shown in Fig. 3 (see panels b and c). In Fig. 4 (panels b and c) one can see again two different intervals (10-50 and 60-130 min) of long quasi-periodic oscillations detected in radio emission at $93 \mathrm{GHz}$. The spectrum for NoRH data $(17 \mathrm{GHz})$ obtained for the same active area also contains two different period intervals: one weak (30-60 min) below the significance level of 95\%, and one powerful (70-130 min) (see Fig. 5, panels b and c).

To compare visible periodic features on the wavelet spectra, we obviously should take into account the time difference between the tracks, because the starts of these tracks were not exactly simultaneous.

However, evidently (Figs. 3, 4) two strong long-period components exist in the wavelet power spectra for $37 \mathrm{GHz}(10-60$ and 80-130 $\mathrm{min}$ ) and $93 \mathrm{GHz}(10-50$ and 60-120 min). In the wavelet power spectrum for $17 \mathrm{GHz}$ data (Fig. 5) one longperiod component exists (80-130 $\mathrm{min}$ ) above the significance level of $95 \%$. Thus we can notice that for the same active region we have found almost the same long quasi-periodic oscillations in the intervals of 60-130 min at three different frequencies that exist at least $800 \mathrm{~min}$ or about $13 \mathrm{~h}$.

In addition, we analyzed radio emission from AR $11084 \mathrm{ob}-$ served on the next day, 2010 July 2, at 17 and 93 GHz. In Fig. 6 

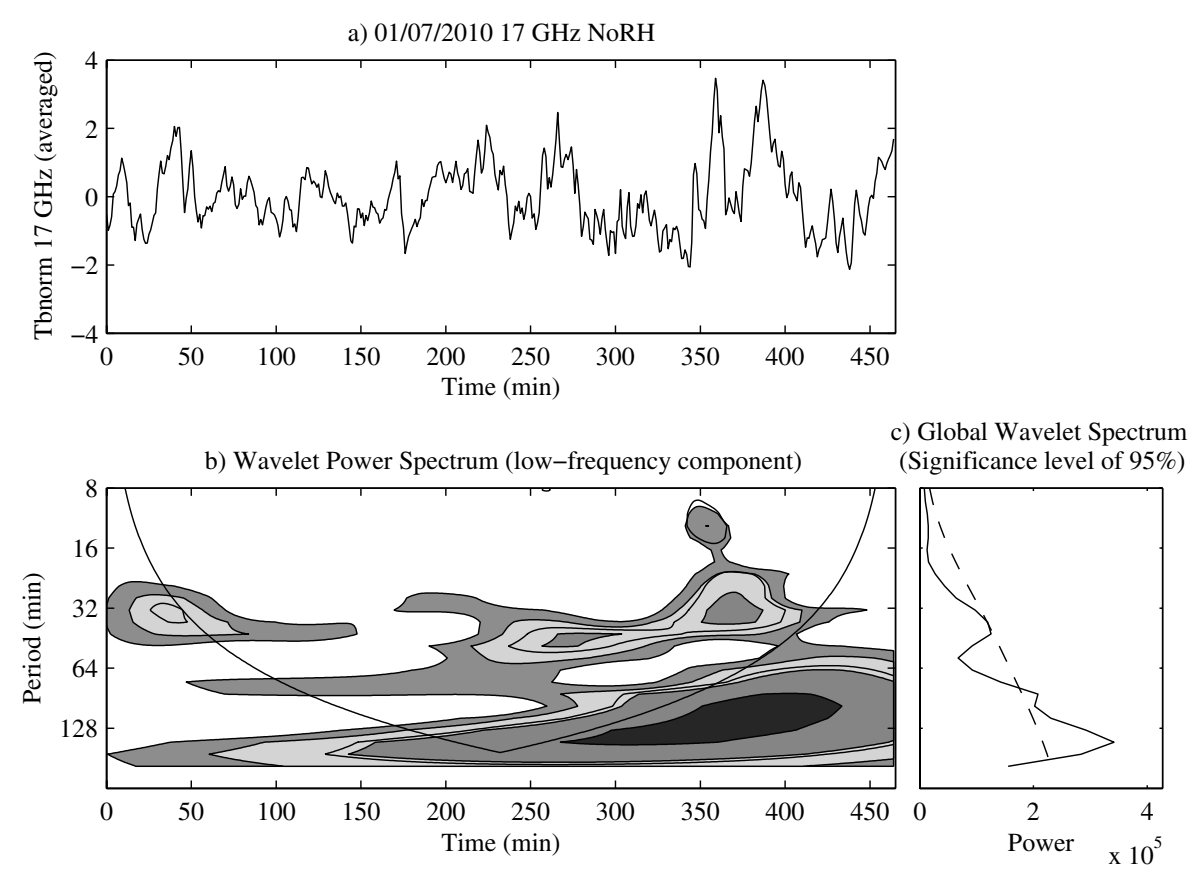

Fig. 5. Same as in Fig. 3 for $17 \mathrm{GHz}$ data on 2010 July 1 for the source AR 11084.

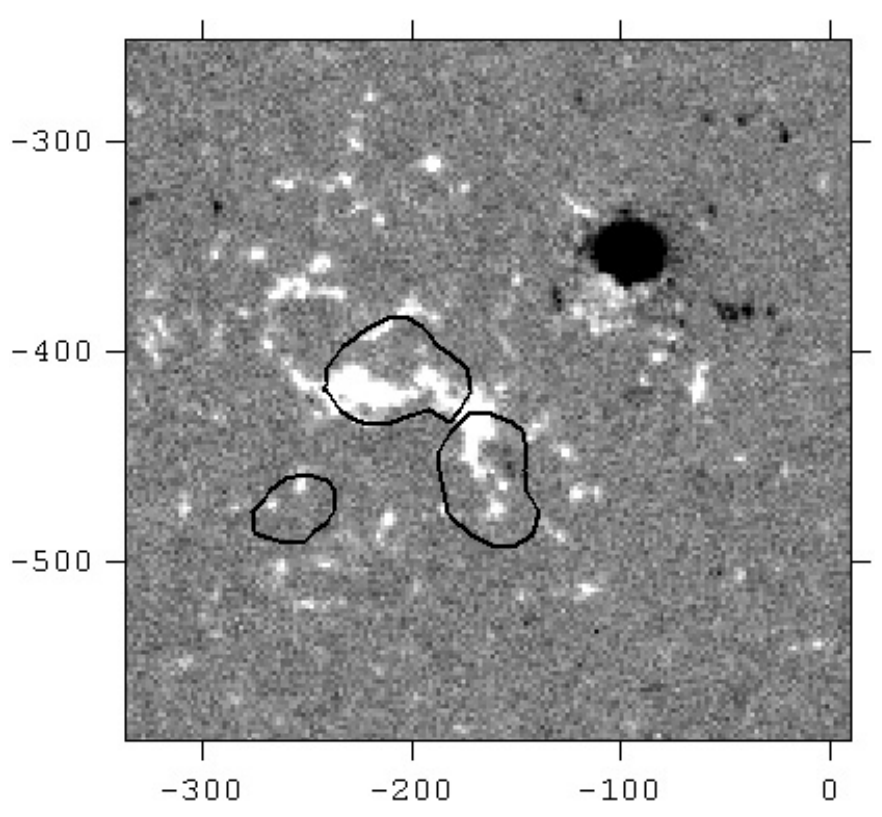

Fig. 6. SOHO/MDI magnetogram (Bmax is about 2000 Gauss) with $17 \mathrm{GHz}$ source contours at the brightness temperature level of $11000 \mathrm{~K}$ on 2010 July 2, AR 11084.

one can see the contours of the $17 \mathrm{GHz}$ source superposed on the magnetogram, coinciding with the distributed positive magnetic field structures.

As on the previous day, two intervals of periods (16-50 and $70-130 \mathrm{~min}$ ) are visible in the wavelet spectrum for $93 \mathrm{GHz}$ data (Fig. 7) and only one interval of periods (60-130 $\mathrm{min}$ ) for $17 \mathrm{GHz}$ data (Fig. 8). The power of these long-period oscillations is above the significance level of $95 \%$. Taking into account these results for AR 11084, we can suggest that the long period (60-130 min) component exists for at least two days.

We analyzed the other data in the same manner.

\section{Discussion and conclusions}

Obviously, one of the main factors to note is the wide beam size of the antennas $\left(2.5^{\prime}\right)$. It is able cover the significant area of the quiet Sun if the active region is small. The size of the radio source above spots could vary from several arc-seconds to arc-minutes. Consequently, it is sometimes difficult to resolve them with the wide beam and separate the flux from the specified active region and from the surrounding quiet area flux.

Oscillations of the quiet Sun regions were detected at the RT-14 Metsähovi at $37 \mathrm{GHz}$ (Kurths et al. 1988). But at present we have no possibility to define the amplitude of quiet Sun oscillations relative to the signal from the active regions at RT-14. During the observational period we provided the tracking of the quiet regions at $93 \mathrm{GHz}$ (RT-7.5) to obtain possible oscillations. The analysis showed oscillations with periods 20-40 min with an amplitude of $5-10 \%$ relative to the signal from the active regions. Evidently, we need more long-lasting observations at the RT-14 and at the RT-7.5 to estimate of the contribution of the quiet Sun to the long quasi-periodic oscillations. Oscillations of the quiet solar regions at $17 \mathrm{GHz}$ have been observed and analyzed, for example, by Chorley et al. (2010). The amplitude of the oscillations was very low compared with the active region oscillations.

The AR 11084 tracks showed powerful oscillations. Using $17 \mathrm{GHz}$ maps obtained with the NoRH, we determined the positions of the radio sources in the SOHO/MDI magnetograms. In all considered cases the radio sources coincided with the distributed magnetic field structures (as shown in Figs. 2 and 6). It means that long periodic radio oscillations were detected from the complex system above the sunspots which consists of magnetic loops and arches with different sizes, heights and orientations. It is important to note that the magnetic field strength of the AR 11084 was 2350 Gauss. In the beginning we proposed that the mechanism responsible for the generation of the oscillations with periods in the range of 60-130 min could be related to the appearance of sub-harmonics of global sunspot oscillations. As result, this conclusion could support the shallow sunspot model suggested by Solov'ev \& Kirichek (2008). 

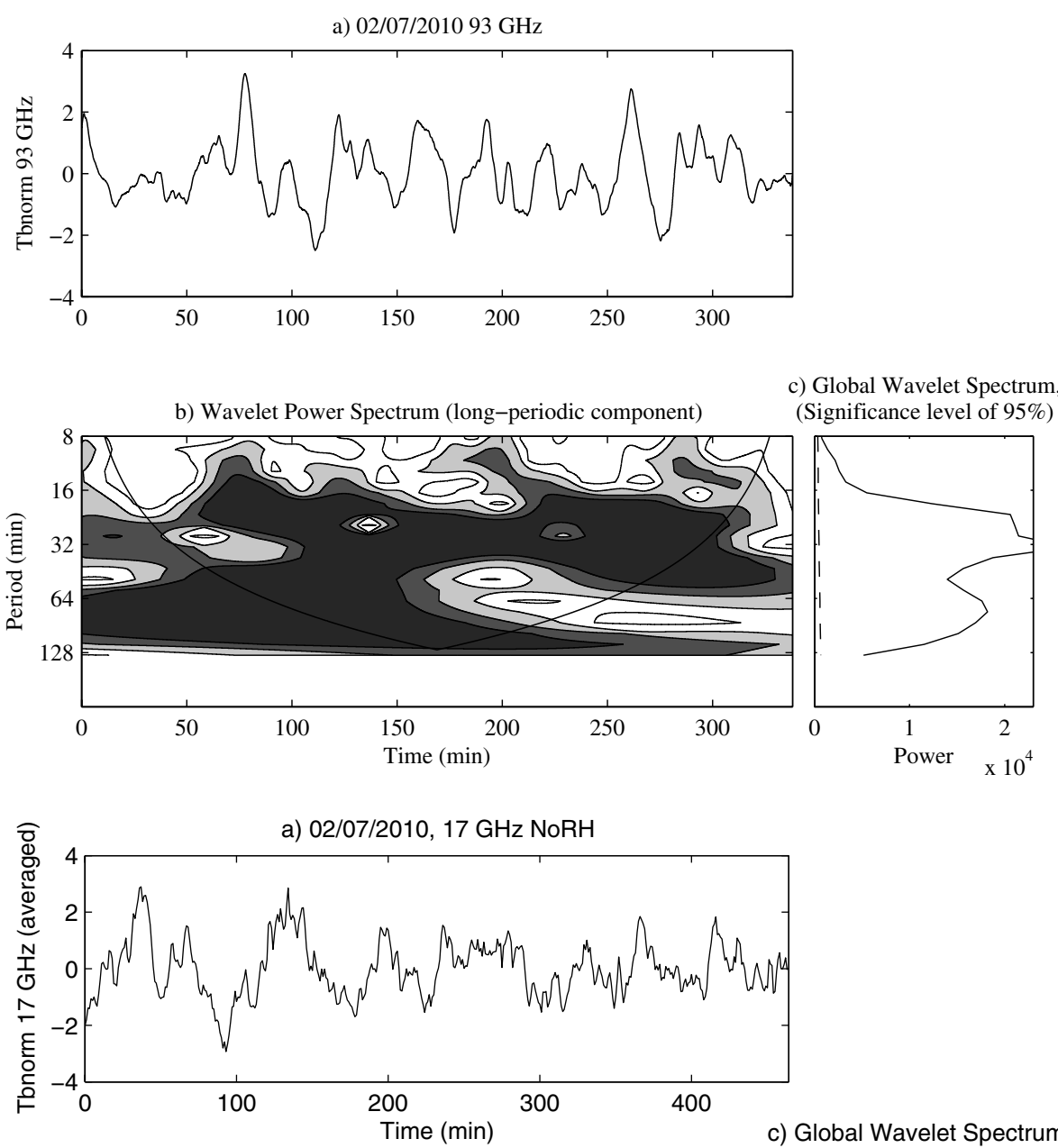

b) Wavelet Power Spectrum (low-frequency component) C) Global Wavelet Spectrum

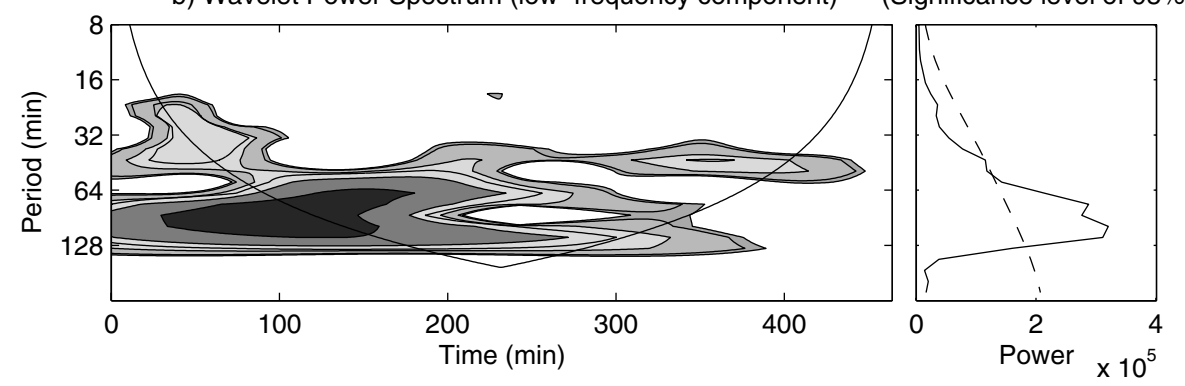

Fig. 7. Wavelet spectrum for $93 \mathrm{GHz}$ data on 2010 July 2 for the AR 11084.

Fig. 8. Wavelet spectrum for $17 \mathrm{GHz}$ data on 2010 July 2 for the AR 11084 .
But the main harmonic of global sunspot oscillations is about 800-900 min (12-15 h) (Efremov et al. 2010), so it is evident that far sub-harmonics of this mode (10-100 min) could be weak and unstable.

But in our case, the periods of about 60-130 min were significantly powerful, relatively stable, they existed at least about two days (for example AR 11084, which was observed during two days).

For this reason we suggested that the oscillations (see Table 1) could be related to the radial mode of sunspot oscillations. This mode appears in quasi-periodic changing of the sunspot area or radial distance between the different parts of the sunspot. One of the possible estimates of period $P$ for the radial mode of sunspot oscillations could be:

$P \simeq \frac{\alpha}{V_{\mathrm{A}}} \simeq 1000 \mathrm{~s}(15 \mathrm{~min})$,

where $\alpha$-is the sunspot radius $10^{9} \mathrm{~cm}$ and $V_{\mathrm{A}}-$ is the Alfvén speed, which is about $10^{6} \mathrm{~cm} / \mathrm{s}$.
But in our case we deal with the magnetic loops, which connect the different parts of the sunspot area (see Fig. 1). Thus, we can estimate the length of the magnetic loops $(\alpha)$ in the interval beginning from 50 to 120 arc-seconds (or $3.5 \times 10^{9}-8.4 \times$ $\left.10^{9} \mathrm{~cm}\right)$. In this case the estimates for period $P$ are in the interval 55-140 min.

This result gives us a possibility to connect the observed long-period fluctuations with the radial mode of global sunspot oscillations. But we note that the expression in formula (1) should not be considered as the possible definition of the type of MHD waves propagated in magnetic loops. Unfortunately, it is not possible with our data to make a qualified decision about the type of MHD waves (Alfvén, slow- or fast magneto-acoustic). Propagation properties of these waves are different. For this reason the applicability of formula (1) is limited and can be used only for a raw estimation.

Obviously, the global oscillations of sunspots could influence the source parameters at chromospheric heights, where 
the complex magnetic field configuration is changing in time. Unfortunately, it is still unclear how these global oscillations of sunspots transmit to the chromosphere-corona heights.

Thus, new long sessions of monitoring observations of active regions in the millimeter range are needed to obtain more qualitative data.

Acknowledgements. We thank Dr. V. G. Nagnibeda and N. A. Topchilo for critical comments and Prof. A. A. Solov'ev for helpful recommendations. We thank Dr. P. H. Scherrer and the SOHO team for providing the SOHO/MDI data. We also thank Prof. K. Shibasaki and the NoRH team for the data access and Prof. E. Valtaoja for the valuable advices and support. Part of work was supported by the Federal Target Program "Research and scientific-pedagogical cadres Innovative Russia" in 2009-2013 years.

\section{References}

Abramov-Maximov, V. E., Gelfreikh, G. B., Kobanov, N. I., Shibasaki, K., \& Chupin, S. A. 2011, Sol. Phys., 270, 175

Bogdan, T. J. 2000, Sol. Phys., 192, 373

Chorley, N., Hnat, B., Nakariakov, V. M., Inglis, A. R., \& Bakunina, I. A. 2010, A\&A, 513, A27
Efremov, V. A., \& Rozanov, B. A. 2001, Physics and Engineering of Millimeter and Submillimeter Waves, Symp. Proc., 796

Efremov, V. I., Parfinenko, L. D., \& Solov'ev, A. A. 2007, Ast. Rep., 51, 401

Efremov, V. I., Parfinenko, L. D., \& Solov'ev, A. A. 2010, Sol. Phys., 267, 279

Gelfreikh, G. B., Grechnev, V., Kosugi, T., \& Shibasaki, K. 1999, Sol. Phys., 185,177

Gelfreikh, G. B., Nagovitsyn, Y. A., \& Nagovitsyna, E. Y. 2006, PASJ, 58, 29

Khodachenko, M. L., Kislyakova, K. G., Zaqarashvili, T. V., et al. 2011, A\&A, 525, A105

Kislyakova, K. G., Zaitsev, V. V., Urpo, S., \& Riehokainen, A. 2011, Ast. Rep, 55,275

Kobrin, M. M., \& Korshunov, A. I. 1972, Sol. Phys., 25, 339

Kosovichev, A. G. 2009, AIPC Proc., 1170, 547

Kurths, J., Aurass, H., Urpo, S., \& Pohjolainen, S. 1988, A\&A, 191, 359

Loukitcheva, M. A., Solanki, S. K., Carlsson, M., \& Stein, R. F. 2004, A\&A, 419, 74

Nagovitsyna, E. Y., \& Nagovitsyn, Y. A. 2001, Astron. Lett., 27, 118

Nagnibeda, V. G., \& Piotrovitch, V. V. 1994, Sol. Phys., 152, 175

Nakajima, H., Nishio, M., Enome, S., et al. 1994, IEEE Proc., 82, 705

Nakariakov, V. M. 2007, Adv. Space Res., 39, 1804

Rozanov, B. A. 1981, Radioelektronika, 24, 3

Solov'ev, A. A., \& Kirichek, E. A. 2008, Astrophys. Bull., 63, 169

Thomas, J. H., Cram, L. E., \& Nye, A. H. 1984, ApJ, 285, 368

Torrence, C., \& Compo, G. P. 1998, Bull. Amer. Meteorolog. Soc., 79, 61

Urpo, S. 1975, Helsinki University of Technology, Radio lab., Report S 73 